\title{
Standardization of Bovine Macrophage Monolayers and Isolation and Culture of Trypanosomes
}

\author{
Omar A Saldarriaga, Jaime I Velásquez*, Jorge E Ossa, Maria T Rugeles/+ \\ Grupo de Inmunovirologia-Biogenesis, Facultad de Medicina *Facultad de MedicinaVeterinaria y de Zootecnia, \\ Universidad de Antioquia, A.A. 1226, Medellín, Colombia
}

\begin{abstract}
We describe a method for culturing over $90 \%$ pure bovine macrophages from peripheral blood mononuclear cells separated with Nycoprep. The cells were cultured for 12 days and then stained with esterase and with anti CD14 to test for purity. The method is reproducible and ensures an adequate number of cells for immunological research. Additionally, we report the unexpected finding of Trypanosoma trypomastigotes in our macrophage cultures from bovines belonging to a geographic area from which no bovine trypanosomes had been reported before.
\end{abstract}

Key words: macrophages - bovine - trypanosomes

Macrophages represent an ubiquitously distributed population of fixed and circulating mononuclear phagocytes that, at least in higher organisms, express a variety of functions other than phagocytosis, including cytokine production, killing of microbes and tumor cells and processing and presentation of antigens to lymphocytes (Qureshi et al. 1996, Cerone et al. 2000). Macrophages exhibit functional, morphological and metabolic diversity depending on the site of origin and on the stage of differentiation or activation. We are interested in studying the phenomenon of natural resistance against Brucella/Salmonella associated with "natural resistance associated macrophage protein" (Nramp1). For this reason and with the aim of in vitro phenotyping cattle for resistance/susceptibility to B. abortus/S. dublin we modified some protocols to come up with a method for macrophage culturing that we find very efficient. Since mature tissue macrophages are not readily available for functional analysis and taking into consideration the key role that these cells play in the immune response, the alternative of using in vitro monocytes-derived macrophages for functional studies is very attractive. Additionally, we report the finding, for the first time in our geographic area (state of Antioquia) of Trypanosoma trypomastigotes appearing in the cultures extra-cellularly, at around 11 days and growing in density until practically destroying the cell monolayer. The promastigote form was never detected.

The detailed procedure is as follows: $300 \mathrm{ml}$ of blood were collected from the jugular vein, from each animal, into citrate fosfate dextrose and adenine (CPDA) antico-

${ }^{+}$Corresponding author. Fax: +57-4-510 6062. E-mail: mtrugel@catios.udea.edu.co

Received 24 April 2002

Accepted 5 December 2002 agulant containing bags (Baxter, Mexico). The sample was then centrifuged at $1000 \mathrm{x}$ g for $25 \mathrm{~min}$. The buffy coat was resuspended in $4 \mathrm{ml}$ of citrate phosphate buffered saline (PBS-citrate) (pH 7.3) and then overlaid on $3 \mathrm{ml}$ of a Nycoprep solution (density = 1077; NYCOMED PHARMA, Oslo, Norway) and centrifuged at $1000 \mathrm{x} g$ for $25 \mathrm{~min}$. Mononuclear cells were collected and washed twice with PBScitrate and resuspended to a concentration of 6-15 x $10^{6}$ cell $/ \mathrm{ml}^{-1}$ in RPMI-1640 medium (SIGMA, Chemical Co., St. Louis, MO) supplemented with $7 \%$ inactivated and filtered autologous serum, 2 mM L-Glutamine (SIGMA), 10 $\mathrm{mM}$ sodium piruvate (SIGMA) and 1\% MEM Non-Essential Amino Acid Solution (100x) (SIGMA); antibiotics were not used. One milliliter of the cell suspension was added to each well of a 24 flat bottom plate (NUNC, Naperville, Illinois) and placed at $37^{\circ} \mathrm{C} / 5 \% \mathrm{CO}_{2}$ (Heraeus Instruments, Hanau, Germany). Twenty four hours later the monolayer was washed twice with pre-warmed $\left(37^{\circ} \mathrm{C}\right)$ PBS and $75 \%$ of the medium was replaced with RPMI supplemented with $10 \%$ autologous serum. At this point, the majority of cells, around $75 \%$, are adherent monocytes that look large and round. On day 8 the monolayer was washed and the non-adherent cells, mostly B-lymphocytes, as tested by flow cytometry, were removed. Again, $75 \%$ of the cell culture medium was replaced with RPMI supplemented with $10 \%$ autologous serum. The most important point to consider in culturing macrophages is the possibility of contamination with other cells such as lymphocytes (Bennett et al. 1992), platelets and red blood cells. However, in our conditions we found that maintaining the culture with contaminant cells, without much manipulation until the second wash (day 8 of culturing) was a more efficient strategy. At this time the non-adherent cells, forming an overlay like film could be easily removed by gently pipetting. The culture medium was replaced every 3 to 5 days and at day 11 the monolayer exhibited $95 \%$ confluence, mainly as a result of cell spreading. At this time the esterase activity was detected using the $\alpha$ naphthyl acetate esterase kit (SIGMA) to find that $95 \%$ of 
cells were positive (Fig. 1). The attached cells could be recovered using cold PBS/10 min and then scraped with cell scrapers. In order to determine the purity of the removed cells they were stained with an FITC labeled anti bovine CD14, Mab (M-M8) (VMRD, Inc. Pullman, WA) and analyzed under flow cytometry; $94.7 \%$ of the cells were positive (Fig. 2). This protocol ensures the recovery of macrophages that can be used in different assays, such as phagocytosis. The addition of autologous serum to the cultures guarantees the presence of growth factors to support the differentiation of monocytes.

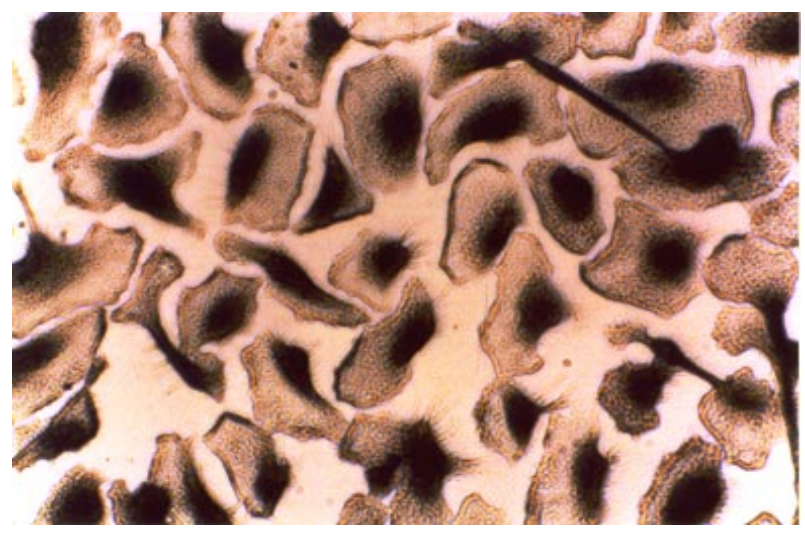

Fig 1: $95 \%$ esterase activity of bovine macrophage monolayer after 11 days of culture.

After 11 days of culture we detected an unusual protozoa contamination of the macrophage cultures (Fig. 3). The parasites were observed swimming freely and the observation was repeated in cultures from different animals from the same farm, along a sampling period of 6 months in which 17 animals were studied and 11 (64.7\%) were found infected. The trypanosomes survived and replicated only if the medium was replaced every 3-5 days. The macrophage monolayers degenerated if the parasites were not removed. Additionally, infected monolayers altered phagocytosis assays, perhaps due to preactivation of the macrophages induced by the parasites. The morphological analysis, the inability to detect the parasites in blood smears, the good health conditions of the animals and the imminent presence of the vector (Tabanus) suggest infection by Trypanosoma theileri. These findings are concordant with Verloo et al. (2000) who found that this trypanosome can actively grow in bovine mononuclear cells cultured in RPMI 1640 medium. Additionally, our observations are in agreement with Ventura et al. (1996) who convincingly state that these parasites are rarely found in peripheral blood smears. It would be interesting to determine what is (are) the particular condition (s) allowing the proliferation of trypomastigotes under these in vitro conditions; it could be the absence of other immune cells like $\mathrm{T}$ lymphocytes or the production of monocyte/macrophage derived factors which could eventually be used by the parasite for its proliferation. Disentangling these interactions may be crucial for further understanding the pathogenesis of trypanosomyasis.

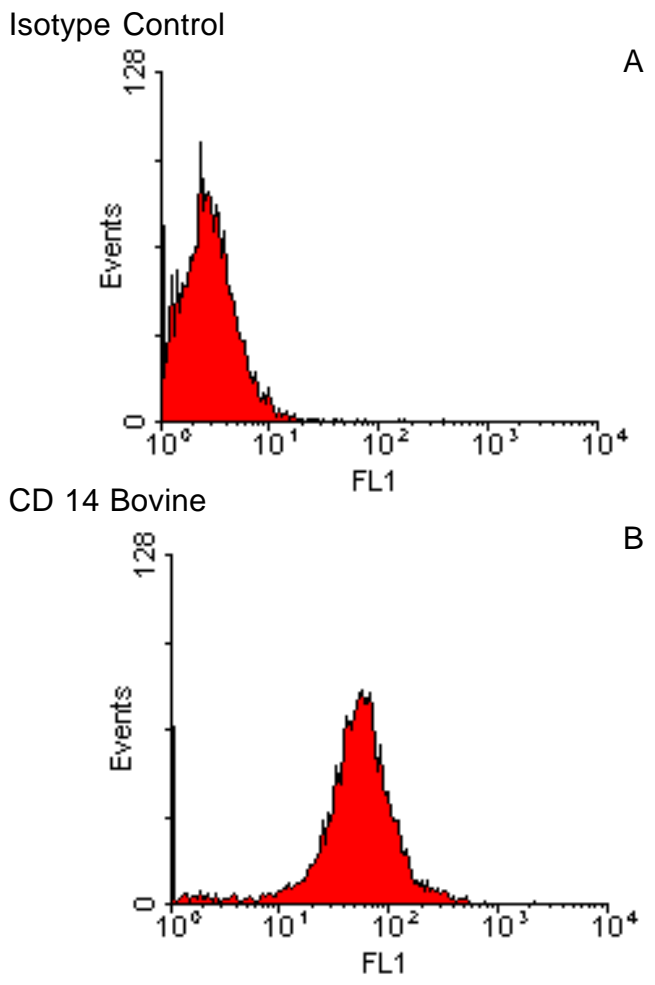

Fig 2: bovine macrophage population after 12 days of culture, analyzed by flow cytometry. A: isotype control; B: $94.7 \%$ of cells in culture express CD14 marker, revealed with FITC-labeled mouse anti-IgG.

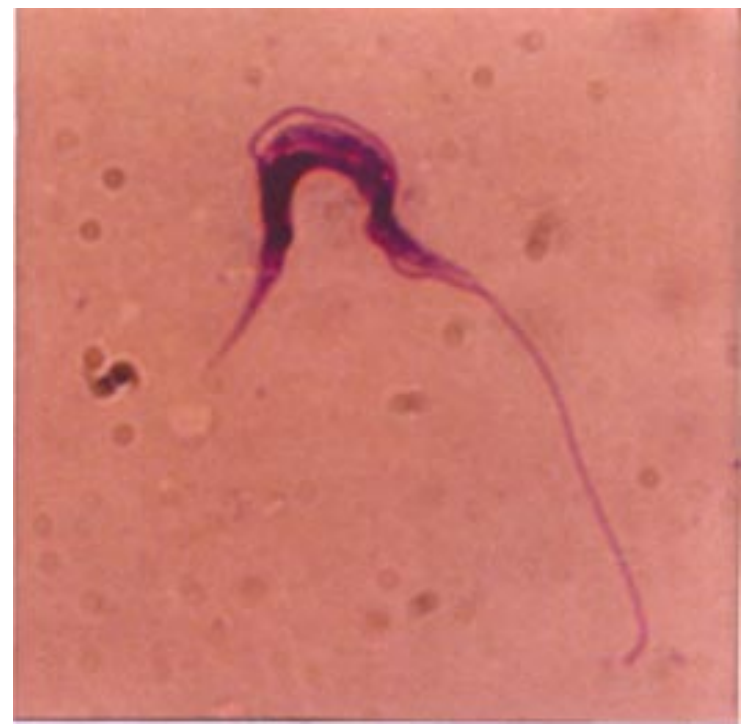

Fig 3: photomicrograph of trypomastigotes found in blood-derived mononuclear cell cultures from different naturally infected cows, from San José del Nus, Antioquia, morphologically characterized as Trypanosoma theileri.

\section{REFERENCES}

Bennett S, Por SB, Stanley ER, Breit SN 1992. Monocyte proliferation in a cytoquine-free, serum free system. J Immunol Meth 153: 201-212. 
Cerone S, Sansinanea A, Streitenberger S, Garcia C, Auza N 2000. Bovine monocyte-derived macrophage function in induced copper deficiency. Gen Phy Biophys 19: 49-58.

Qureshi T, Templeton JW, Adams LG 1996. Intracellular survival of Brucella abortus, Mycobacterium bovis BCG, Salmonella dublin, and Salmonella typhimurium in macrophages from cattle genetically resistant to Brucella abortus. Vet Immunol Immunopathol 50: 55-65.
Ventura RM, Rodríguez AC, Dell'Porto A, Campaner M, Takeda G, Teixeira MM 1996. Molecular tools for the identification and characterization of Trypanosoma (Megatrypanum) spp. and Trypanosoma (Trypanozoon) evansi. En: http:// memorias.ioc.fiocruz.br/96caxambu/rt1.html Verloo D, Brandt J, Meirvenne NV, Buscher P 2000. Comparative in vitro isolation of Trypanosoma theileri from cattle in Belgium. Vet Parasitol 89: 129-132. 